\title{
On passive characterization of aggregated traffic in wireless networks
}

\author{
Anna Chaltseva and Evgeny Osipov \\ Department of Computer Science Electrical and Space Engineering \\ Luleå University of Technology, \\ 97187 Luleå, Sweden, \\ \{Anna.Chaltseva, Evgeny.Osipov\}eltu.se
}

\begin{abstract}
We present a practical measurement-based characterization of the aggregated traffic on microseconds time scale in wireless networks. The model allows estimating the channel utilization for the period of time required to transmit data structures of different sizes (short control frames and a data packet of the maximum size). The presented model opens a possibility to mitigate the effect of interferences in the network by optimizing the communication parameters of the MAC layer (e.g. the size of contention window, retransmission strategy, etc.) for the forthcoming transmission. The article discusses issues and challenges associated with the PHY-layer characterization of the network state.
\end{abstract}

Keywords: Aggregated traffic, RSSI, modeling

\section{Introduction}

Interference from external sources (noise) as well as interferences caused by distant communications on the same radio channel are the main reasons for the unstable performance in wireless networks in general and those built upon the IEEE 802.11 standard in particular. The "h" extension of the IEEE 802.11 standard [1] defines a Dynamic Frequency Selection (DFS) mechanism. The main idea of DFS is to reduce the interferences between wireless nodes by estimating the current utilization of the available channels based on RSSI (Received Signal Strength Indication) statistics and assuming that the estimated channel state will persist in a short-term future. In this article we present the results of a preliminary investigation of a possibility of using the statistics of the received signal strength not only to conclude about the channel utilization at the time of taking measurements but also predicting the channel utilization in the short-term future, further conceptualized in Figure 1(a). If the approach is successful the predicted in this waychannel utilization could be used to adjust the parameters of the MAC layer (e.g. size of contention window, retransmission strategy, etc.), so to minimize the packet collision probability. This optimization process falls however outside the scope of this work and will be reported elsewhere.

Our major results are twofold. On the positive side we show that the statistics collected at the physical layer do not behave randomly and it is valid to use this information for characterization of the aggregated traffic in the vicinity of a wireless transmitter. For 


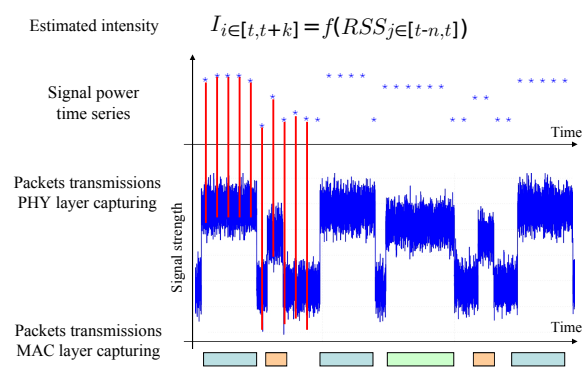

(a) Overall concept

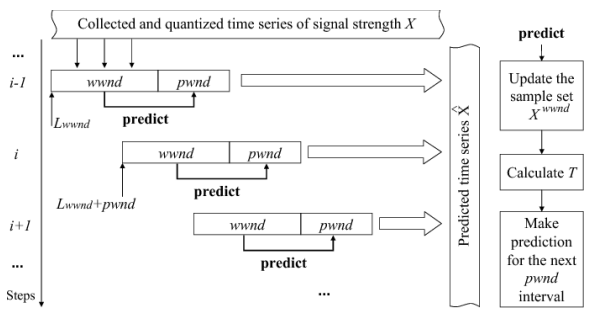

(b) The modeling process (see details in Section 3.3).

Fig. 1. Assessment of the quality and accuracy of the model.

this purpose we propose a Markov-based model, which allows to predict the channel utilization on micro- and millisecond's time scale. While showing the feasibility of the micro-scale traffic characterization we conclude that more efforts should be spend to increase the accuracy of the prediction as well as developing mechanisms for using this information to improve the performance of next generation cognitive MAC protocols.

The most related to the topic of this article works are [2,3]. The authors in [2] use the autocorrelation function to predict the channel state ("free" or "busy"). In this work we show that the autocorrelation function cannot provide a conclusive picture in the case of mixed traffic under high load. In [3] the authors analytically model the instantaneous spectrum availability for a system with multiple channel using partially observable Markov decision process. This work presents decentralized cognitive MAC which allows the maximization of the overall network throughput. The results of our work could be considered in some extend as a practical compliment to the later approach since we build an empirical estimator of the instantaneous (plus several milliseconds in the future) channel state.

The article is organized as follows. Section 2 presents the research methodology. The passive estimation of the traffic intensity including the description of the experiments, data analysis, modeling, and the assessment of the accuracy is presented in Section 3, which is the main section of this article. Section 4 concludes the article.

\section{Methodology}

The main hypothesis of our work is that it is possible to derive a PHY-layer characterization of the aggregated traffic on a wireless link by statistical analysis of time series of the received signal strength. Our methodology for verification of the hypothesis consists of three phases: data gathering; randomness and correlation analysis; and modeling and assessment.

Data gathering: All data for further analysis and modeling were obtained in a controllable manner in a radio isolated chamber. We experimented with traffic of different intensities and used a spectrum analyzer to accurately record the signal strength time 
series with microsecond's sampling time. The detailed description of the experiments follows in Section 3.1.

Randomness and correlation analysis: In this phase we firstly examine a statistical dependence in the recorded time series. In other words whether we can use the physical layer's statistics for characterization of the channel utilization. The results of the twosample Kolmogorov-Smirnov test (presented in Section 3.2) allowed us to proceed with the analysis of nature of the statistical dependence by studying the correlation structure of the series described in the same section.

Modeling and assessment: Finally, we build a two-state Markov model of the channel occupancy and use it to estimate channel utilization in time domain during a time interval chosen with reference to the transmission time of data structures of different length (e.g. short control frames and maximum size of a data packet). The rationale for doing this step is simple, if we are able to correctly predict the channel utilization on packet -ransmission time scale we may further use this result to optimize the transmissions of the pending packets.

\section{Passive estimation of aggregated traffic intensity using PHY-layer statistics}

In this section we develop our hypothesis of deriving PHY-layer characterization of the aggregated traffic. The subsections below describe the details of data gathering, randomness and correlation analysis as well as present the constructed model and the results of its accuracy assessment.

\subsection{Test-bed Experiments and Data Gathering}

The time series of the received signal strength were measured during a set of experiments performed on a wireless test-bed network located inside an isolated $6 \times 3$ meters chamber. The walls of the chamber are non-reflecting surfaces preventing multipath propagation. The wireless test-bed consists of four computers equipped with IEEE 802.11 abgn interfaces, located in the transmission range of each other. All computers are running Linux operating system (kernel 2.6.32). The transmitted signal power was set to $18 \mathrm{dBm}$, the testbed operated on channel $4(2427 \mathrm{MHz})$. On the MAC layer the Maximum Contention Window is 1023, the short slot time is 9 us, SIFS interval is 10 us, and the short preamble is 72 bits.

The received signal strength time series were recorded using spectrum analyzer Agilent E4440A. The recorded raw signal was sampled with $1 \mathrm{MHz}$ frequency. Later during the analysis phase we increased the sampling interval by trimming out the original set. We quantized the recorded signals into two levels. All samples with the signal power less than $-87 \mathrm{dBm}$ (the received sensitivity of the used wireless adapter) were assigned a value of 0 (zero). All measurements above this threshold were assigned a value of 1 .

Traffic flows: In total 13 experiments with one, two, three, and four concurrent data sessions were performed. For further discussions we sort all experiments into three groups depending on the aggregated load (low, medium, and high). 
The low traffic was generated by single UDP or TCP flows, the medium traffic was generated by two and three concurrently running UDP and TCP flows in different combinations, the high traffic was generated by four concurrently running UDP and TCP flows in different combinations. In all cases nodes were configured with static routing information in order to eliminate the disturbance caused by the routing traffic. In all experiments the payload size was chosen so to fit the maximum transfer unit of 1460 Bytes. In the case of UDP traffic we experimented with two traffic generation rates: 100 $\mathrm{Kb} / \mathrm{s}$ and $11 \mathrm{Mb} / \mathrm{s}$, to study both the unsaturated and saturated cases. The duration of each experiment was 10 seconds. To remove transient effects, only the last 2.5 seconds of the recorded signal series were used for the analysis.

\subsection{Randomness and Correlation Analysis}

Denote $X^{C}=\left\{x_{i}^{C}\right\}$ the recorded continuous time series. Let $X^{R}=\left\{x_{i}^{R}\right\}$ denote a reference random time series obtained by randomly shuffling the original set $X^{C}$. In order to verify whether there is a statistical dependency in the original time series we performed the analysis of increments dependence [4]. The increments of time series were obtained for both $X^{C}$ and $X^{R}$ as $\Delta x_{i}^{C}=x_{i}^{C}-x_{i-1}^{C}$ and $\Delta x_{i}^{R}=x_{i}^{R}-x_{i-1}^{R}$ correspondingly.

In order to check the hypothesis that the recorded time series of the signal strength have a statistical dependency, the two-sample Kolmogorov-Smirnov test [5] was performed. This test compares the distributions of the values in the two given sets (the original and the reference time series). The null hypothesis is that the sets are from the same continuous distributions, accordingly, the alternative hypothesis is that the sets are from different distributions. The outcome of Kolmogorov-Smirnov allows the rejection of the null hypothesis with $1 \%$ significance level, i.e. there is a statistical dependency in the recorded time series. This conclusion allows us to proceed with the analysis.

The analysis of the autocorrelation function for the time series showed very rapid decay of curves, which indicates that all observed processes have short-range dependence. The graphs are omitted here due to the limited space, the reader is referred to [6].

\subsection{Modeling and assessment}

Our approach towards modeling is illustrated in Figure 1(b). A two-state Markov model is constructed using data collected during time interval called working window and denoted as wwnd. During the course of this work we experimented with different durations of $w w n d$. It appeared that the size of $w w n d$ does not significantly affect the accuracy of the prediction. The results presented in this article are obtained using $w w n d=0.5 \mathrm{~s}$. Denote $X=\left\{x_{i}\right\}, x_{i} \in[0 ; 1]$ the post processed and quantized time series of $X^{C}$ (See Section 3.1).

The constructed model is then used to predict the presence or absence of the signal during the immediately following time interval called prediction window and denoted as pwnd. The size of pwnd is chosen with a reference to the time of transmitting a data structure of certain length with a given transmission rate on the physical layer. We choose two values of pwnd in order to illustrate our reasoning: one equals the 
time it takes to transmit the shortest data structure (RTS frame) with the rate $1 \mathrm{Mb} / \mathrm{s}$ : pwnd $=200$ microseconds. The other value equals the time it takes to transmit the maximum size packet (1460 Bytes) with the highest transmission rate $11 \mathrm{Mb} / \mathrm{s}$ in our case: $p w n d=1.5$ milliseconds. The rationale for choosing these values stems from the goal of this work - we want to optimize the performance of the MAC protocol prior of the transmission of a pending packet.

Two-state Markov model over $w w n d$ : Denote $X^{\text {wwnd }}$ a subset of the measured and quantized time series of the received signal strength $X$ of size $w w n d$ expressed in number of samples. Then $x_{i}^{w w n d}$ denotes the measured and quantized signal strength at sample time $i$. The Markov model describes the state of the channel at a particular sampling step $i+1$ based on the current state at the step $i$. The model is defined by a transition probability matrix $T$ as follows:

$$
T=\left(\begin{array}{l}
P\left(x_{i+1}^{w w n d}=0 \mid x_{i}^{w w n d}=0\right) P\left(x_{i+1}^{w w n d}=1 \mid x_{i}^{w w n d}=0\right) \\
P\left(x_{i+1}^{w w n d}=0 \mid x_{i}^{w w n d}=1\right) P\left(x_{i+1}^{w w n d}=1 \mid x_{i}^{w w n d}=1\right)
\end{array}\right)
$$

where $P$ is an empirical conditional probability calculated over wwnd number of samples.

When matrix $T$ is calculated and the prediction of the channel utilization (as described below) is done we shift the working window on the set of original time series $X$ to pwnd samples in the direction of time increase. This moves us to the next iteration of the modeling and prediction process, which is summarized in Figure 1(b).

Prediction procedure over pwnd: The goal of the prediction process is to generate time series $X^{\text {pwnd }}$ of the predicted signal presence. Thus $x_{i}^{p w n d}=1$ indicates the presence of the signal above the receiver sensitivity threshold while the value of 0 indicates an absence of the signal at sample time $i$. The probabilities that during $i$ th position of pwnd there will be transmission or not are taken from the matrix $T$ depending on the channel state at time $i-1$. After the probabilities are determined for position $i$ of pwnd we generate an actual value ( 1 or 0 ) using conventional technique for generating random numbers from a given distribution. This procedure is then repeated for all positions inside the pwnd.

\subsection{Assessment of the quality and accuracy of the model}

The quality of the model was evaluated by the analysis of the model's performance using normalized Kullback-Leibler divergence. The Kullback-Leibler divergence is a non-symmetric measure of the distance or the relative entropy between two probability distributions $\operatorname{Pr}[X]$ and $\operatorname{Pr}[\hat{X}]$ [7]. This statistical metric (1) is used to measure how the distribution of the set produced by a stochastic model $(\operatorname{Pr}[\hat{X}])$ is different from the distribution of the original stochastic process $\operatorname{Pr}[X]$.

$$
D_{K L}(\operatorname{Pr}[X] \| \operatorname{Pr}[\hat{X}])=\sum_{i} \operatorname{Pr}[X]_{i} * \log \frac{\operatorname{Pr}[X]_{i}}{\operatorname{Pr}[\hat{X}]_{i}}
$$

The smaller is the value of $D_{K L}(\operatorname{Pr}[X] \| \operatorname{Pr}[\hat{X}])$ the closer the distributions $\operatorname{Pr}[X]$ and $\operatorname{Pr}[\hat{X}]$ are. In the case when $D_{K L}(\operatorname{Pr}[X] \| \operatorname{Pr}[\hat{X}])=0$ the two distributions are 


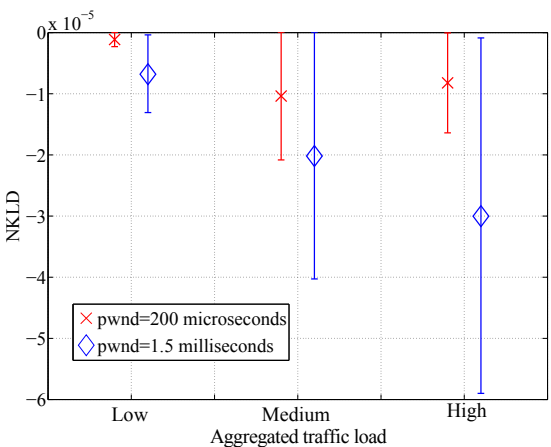

(a) NKLD for the proposed model.

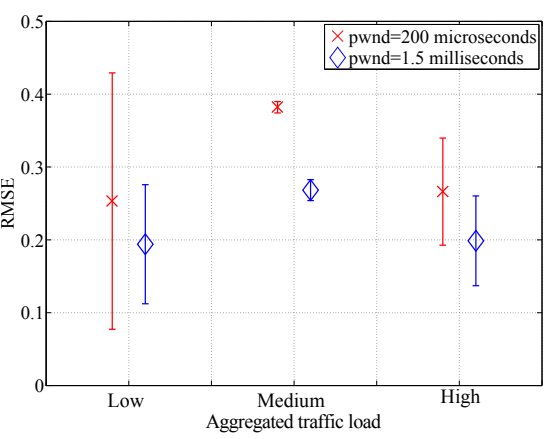

(b) The proposed model accuracy.

Fig. 2. Assessment of the quality and accuracy of the model.

identical. To calculate the normalized Kullback-Leibler distance the following formula was used: $\bar{D}_{K L}(\operatorname{Pr}[X] \| \operatorname{Pr}[\hat{X}])=\frac{D_{K L}(\operatorname{Pr}[X] \| \operatorname{Pr}[\hat{X}])}{H(\operatorname{Pr}[X])}$ where $H(\operatorname{Pr}[X])$ is the entropy of a random variable with the probability mass function $\operatorname{Pr}[X] . H(\operatorname{Pr}[X])=$ $\sum_{i} \operatorname{Pr}[X]_{i} * \log \frac{1}{\operatorname{Pr}[X]_{i}}$.

Figure 2(a) shows the correspondent graphs of $\bar{D}_{K L}$ for the proposed model. We conclude that the model has satisfactory quality since the distance between the probability distributions of the measured time series and the predicted ones is in the oder $10^{-5}$.

Model Accuracy: The accuracy of the model was evaluated with respect to its ability to predict the channel utilization over one pwnd interval, denoted as $\xi^{\text {pwnd }}$ (2). The results are presented in Figure 2(b).

$$
\xi_{j}^{p w n d}=\frac{\sum_{i=1}^{p w n d} x_{i}^{p w n d}}{p w n d}
$$

The predicted utilization over one pwnd interval is denoted as $\hat{\xi}_{j}^{\text {pwnd }}=\frac{\sum_{i=1}^{p w n d} \hat{x}_{i}^{\text {pwnd }}}{p w n d}$, where $j \in[1, N]$ and $N$ is the number of pwnd intervals in $X$ and $\hat{X}$.

As the result of calculation of $\xi$ and $\hat{\xi}$ over the original and predicted time series we obtain two sets of utilization $\Xi=\left\{\xi_{j}^{\text {pwnd }}\right\}$ and $\hat{\Xi}=\left\{\hat{\xi}_{j}^{\text {pwnd }}\right\}$ of measured and predicted utilizations on pwnd chunks of the time series $X$ and $\hat{X}$ correspondingly. $\hat{\Xi}(3)$.

We use the root-mean-square error metric to assess the differences between $\Xi$ and

$$
R M S E=\sqrt{\frac{\sum_{i=1}^{N}\left(\xi_{i}-\hat{\xi}_{i}\right)^{2}}{N}}
$$

Figure 2(b) illustrates the accuracy of the model for different aggregated traffic loads and different values of pwnd. The plot is obtained by assessing the model's accuracy 


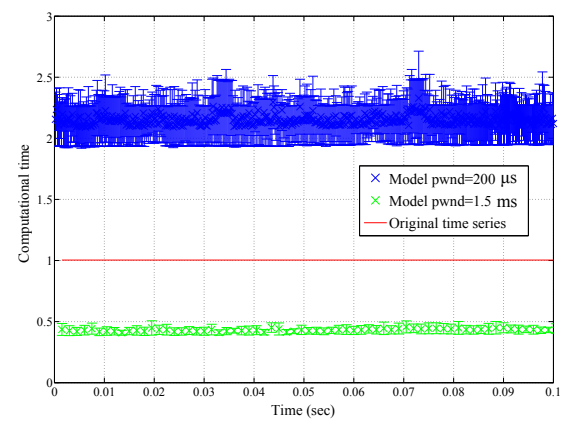

Fig. 3. The computation time of the model.

using three different initial positions for $w w n d$ and correspondingly pwnd in the original set of time series $X$. From Figure 3 we observe that the accuracy of the model is substantially lower for the short pwnd (200 microseconds). Although for some parts of the traces with low traffic intensity the model introduced $10 \%$ error, the average error for all traffic loads ranges between 0.25 and 0.4 . On the other hand for the larger pwnd the average value never exceeds 0.3 for all traffic loads. In particular in the case of high traffic load our models shows 0.2 prediction error.

Computation time: In order to assess the computation time of the model we timed the execution of operations for constructing the transition matrix and the prediction procedure for different values of $p w n d$. The time measurements were performed on Lenovo ThinkPad T61 computer with Intel T7300 Core 2 Duo processor, 2GB RAM and running Ubuntu 10.04 LTS operating system. Figure 3 plots the results of the measurements normalized to the duration of corresponding pwnd. From the figure one could immediately observe that the choice of pwnd size is essential. The computation time of the model is almost twice higher than the duration of the smallest pwnd (200 microseconds) . On the other hand it is twice less than the duration of the larger pwnd (1.5 milliseconds).

\section{Conclusions}

In this article we presented a practical measurement-based model of the channel utilization on microsecond's time scale for wireless networks. The model allows estimating the utilization for the period of time required to transmit data structures of different sizes (short control frames and a data packet of the maximum size). The resulting model opens a possibility to mitigate the effect of interferences in the network by optimizing the parameters of the MAC layer for the forthcoming transmission based on the predicted channel utilization based on short-term historical data. The presented model is based on the collected statistic in the wireless test-bed network located inside an isolated chamber and there is clearly a need in additional experimental work in order to validate the model applicability and accuracy in real settings.

Our major conclusion is twofold. Firstly, more efforts should be spend to increase the accuracy of prediction by using more sophisticated models as well as choosing the 
appropriate dimensions of the working and prediction windows. Here one should make a trade-off between the prediction accuracy and the computation time of the model. Secondly, we foresee that on micro- or millisecond's time scale even the best models would introduce significant error to the predicted channel utilization. It is unrealistic to expect that aggregated traffic could be very accurately characterized solely based on samples of radio signal. One, howeve, still may use this information in more sophisticated cross-layer decision mechanisms. Further development of these issues is a subject for our ongoing and future investigations.

\section{References}

1. "Ieee standard for information technology - telecommunications and information exchange between systems - local and metropolitan networks - specific requirements - part 11: Wireless lan medium access control (mac) and physical layer (phy) specifications - spectrum and transmit power management extensions in the $5 \mathrm{ghz}$ band in europe," 2003.

2. S. Mangold and Z. Zhong, "Spectrum agile radio: Detecting spectrum opportunities," in International Symposium on Advanced Radio Technologies ISART, Boulder CO, USA, Mar 2004, p. 5. [Online]. Available: http://www.comnets.rwth-aachen.de

3. Q. Zhao, L. Tong, and A. Swami, "Decentralized cognitive mac for dynamic spectrum access," in New Frontiers in Dynamic Spectrum Access Networks, 2005. DySPAN 2005. 2005 First IEEE International Symposium on, nov. 2005, pp. $224-232$.

4. A. Kovalevskii, "Dependence of increment in time series via large deviations," in Science and Technology, 2003. Proceedings KORUS 2003. The 7th Korea-Russia International Symposium on, vol. 3, july 2003, pp. $262-267$ vol.3.

5. M. A. Stephens, "Use of the kolmogorov-smirnov, cramer-von mises and related statistics without extensive tables," Journal of the Royal Statistical Society. Series $B$ (Methodological), vol. 32, no. 1, pp. pp. 115-122, 1970. [Online]. Available: http://www.jstor.org/stable/2984408

6. A. Chaltseva, "Network state estimation in wireless multihop networks," 2012, licentiate thesis. [Online]. Available: http://pure.ltu.se/portal/files/35972873/Anna_C._Jaquier.Komplett.pdf

7. T. Cover and J. Thomas, Elements of information theory, ser. Wiley Series in Telecommunications and Signal Processing. Wiley-Interscience, 2006. [Online]. Available: http://books.google.com/books?id=EuhBluW31hsC 